\title{
Renal arterial resistive index in Egyptian patients with lupus nephritis: Correlation with disease activity and biopsy parameters
}

\author{
Hala El-Wakil ${ }^{1}$, Eman KhaliI ${ }^{2 \star}$, Dalia Maharem ${ }^{2}$, El-Said Mahena ${ }^{3}$ and Mohamed Hasab ${ }^{2}$ \\ ${ }^{1}$ Department of Internal Medicine, Faculty of Medicine, Alexandria University, Egypt. \\ ${ }^{2}$ Department of Internal Medicine, Medical Research Institute, Alexandria University, Egypt. \\ ${ }^{3}$ Department of Radiology, Medical Research Institute, Alexandria University, Egypt.
}

Accepted 12 March, 2018

\begin{abstract}
Lupus nephritis (LN) affects up to $60 \%$ of patients with systemic lupus erythematosus (SLE), either as the initial manifestation or during the disease course. Moreover, LN has a negative impact on survival of SLE patients. Accordingly, it is mandatory to identify specific and feasible markers able to guide clinicians towards the adequate therapeutic option in LN patients. The aim of this work was to evaluate the predictive value of renal resistance index (RRI), measured by Doppler Sonography in comparison with disease activity score, serologic and biopsy parameters in patients with LN. This study was carried out on forty three SLE patients, they were categorized into two groups: Group I included thirty three patients with LN and Group II included ten patients without LN and Group III included ten healthy subjects of matched age and sex as control group. All were subjected to history taking, clinical examination, assessment of disease activity by SLEDAI, laboratory investigations including FBG, blood urea, serum creatinine, serum albumin, CBC, ESR, CRP, complete urine analysis, 24 hour urine protein, eGFR, serum ANA, anti ds-DNA titre, C3, C4 and renal Doppler with measurement of RRI. Renal biopsy was done for those with $L N$. The mean value of RRI was statistically significantly higher in group I than that of group II and group III. Out of 33 cases of LN cases, 6 patients had $\mathrm{RRI}$ of 0.7 and above giving a percentage of $18.18 \%$. LN patients with RRI higher than 0.7 had statistically significant higher age, mean serum creatinine and blood urea levels and a lower eGFR, higher chronicity index of renal biopsy. RRI is of clinical significance in predicting the chronicity index of renal biopsy which is a major determinant of renal outcome so it is useful as non invasive technique to evaluate chronicity in patients with LN, therefore justifying aggressive immune suppression but further follow-up studies are needed to evaluate its role in predicting response to treatment.
\end{abstract}

Keywords: Systemic lupus erythematosus, lupus nephritis, renal resistive index.

*Corresponding author. E-mail: d_emansalahkhalil@yahoo.com.

\section{INTRODUCTION}

Systemic lupus erythematosus (SLE) is a chronic inflammatory multisystem disease mainly affecting women of childbearing age. It is characterized by a very large spectrum of clinical manifestations accompanied by prototypic abnormalities of the immune system with unpredictable flares and remissions (Lawrence et al., 1998). Many of its clinical manifestations are secondary to immune complex deposition in capillaries of visceral structures or to autoantibody mediated destruction of host cells. The severity may vary from a mild episodic disorder to a rapidly fulminating life threatening illness (Edworthy, 2001).

The reported prevalence of SLE in the population is 20 to 150 cases per 100,000 . Due to improved detection of mild disease, the incidence nearly tripled in the last 40 years of the $20^{\text {th }}$ century (Uramoto et al., 1999) SLE is 
usually a post pubertal disease, with onset of clinical symptoms between 20 and 30 yrs with a female to male ratio 9:1 (Uramoto et al., 1999).

The exact patho-etiology of SLE remains elusive. An extremely multi-factorial interaction among genetic and environmental components is probably involved (Moc and Lau, 2003). These factors lead to an irreversible break in immunological tolerance manifested by immune responses against endogenous nuclear antigens (Bertsias et al., 2012). According to the SLICC rule for the classification of SLE, the patient must satisfy at least 4 criteria, including at least one clinical criterion and one immunologic criterion OR the patient must have biopsy proven lupus nephritis in the presence of antinuclear antibodies or anti-double-stranded DNA antibodies (Petri et al., 2012).

The kidney is the most commonly involved visceral organ in SLE. Lupus nephritis (LN) is one of the most serious complications of SLE since it is associated with significant morbidity and mortality. In the United States, approximately $35 \%$ of adults with SLE have clinical evidence of nephritis at the time of diagnosis; with an estimated total of 50 to $60 \%$ developing nephritis during the first 10 years of disease (Lee et al., 2010; Hahn et al., 2012; Corapi et al., 2015).

Lupus nephritis is diagnosed by either the presence of proteinuria ( $>0.5 \mathrm{~g} /$ day), active urinary sediment (with red blood cell, granular, tubular and/or mixed casts), or an unexplained rise in serum creatinine. A kidney biopsy is the gold standard to diagnose $L N$ as it provides information regarding the pattern and severity of renal involvement as well as the stage, activity and chronicity. These are all important considerations influencing treatment decisions (Corapi et al., 2015).

Renal resistive index (RRI) is commonly used as an index of intra-renal arterial resistance; it increases in various kidney diseases. It has been used in a variety of clinical settings such as the assessment of chronic renal allograft rejection, parenchymal nephropathies, acute kidney injury, detection and management of renal artery stenosis, evaluation of progression risk in chronic kidney disease (CKD), diabetic nephropathy, differential diagnosis in acute and chronic obstructive renal disease, cirrhosis and hepatorenal syndrome and as a predictor of renal and overall outcome in the critically ill patient (Radermacher et al., 2003; Crutchley et al., 2009; Radermacher et al., 2002; Granata et al., 2009; Viazzi et al., 2014; Lubas et al., 2014; Le Dorze et al., 2012).

Few studies have been carried out on patients affected by systemic vasculitis to evaluate the association between the anatomo-pathological findings and Doppler ultrasound parameters (Platt et al., 1990; Granata et al., 2014). Interestingly, the morphological changes of the Doppler waves found in these patients are similar to those of patients affected by renal microangiopathy related to haemolytic-uraemic syndrome (Patriquin et al., 1989).

$$
\text { Although Doppler sonography clearly does not }
$$

substitute for renal biopsy, several studies have suggested that Doppler sonography might aid in the management of established renal disease (Hanamura et al., 2012; Sugiura and Wada, 2011; Parolini et al., 2009). In a series published by Patriquin et al. (1989), Doppler sonography could predict renal recovery from hemolytic uremic syndrome before clinical improvement. Similarly, the RRI was thought to correlate well with renal involvement in patients with progressive systemic sclerosis (Mostbeck et al., 1991). Previous data have revealed a significant correlation between the RRI value and renal functional parameters (that is, creatinine and urea nitrogen blood levels) and/or histologic modifications, such as glomerulosclerosis, tubulointerstitial damage and vascular lesions (Chen et al., 2014; Ikee et al., 2005; Aikimbaev et al., 2001).

Use of conventional ultrasound has been previously reported in the evaluation of lupus nephritis (Stanley et al., 1984). Abnormalities in renal size, cortical echogenicity have been reported as they have with many forms of renal disease (Platt et al., 1997).

\section{Aim of study}

The goal of our study is to evaluate the predictive value of RRI by Doppler US as compared to disease activity scores, serological and biopsy parameters in patients with lupus nephritis.

\section{SUBJECTS AND METHODS}

This study included forty three patients fulfilling Systematic Lupus International Collaborating Clinics (SLICC) Classification 2012 Criteria for diagnosing SLE disease (Petri et al., 2012). Patients were selected from the Nephrology ward and outpatient clinic in the Medical Research Institute, Alexandria University from March 2015 till March 2016.

Patients were categorized into three groups:

Group I: Thirty three patients with lupus nephritis diagnosed by the presence of proteinuria greater than $0.5 \mathrm{~g} /$ day or abnormal urinary casts (Jane and David, 2003).

Group II: Ten SLE patients without lupus nephritis.

Group III: Ten healthy subjects of matched age and sex as the patients as control group.

Patients with connective tissue diseases other than SLE, diabetes mellitus, hypertensive nephropathy, viral hepatitis B or C, active infections or malignancies, obstructive nephropathy, drug or toxic induced nephropathy and those with end stage renal disease were excluded from this study.

All patients were subjected to the following:

i) Detailed history taking.

ii) Thorough systemic physical examination.

iii) Assessment of disease activity by applying the score of SLE disease activity index (SLEDAI) (Isenberg et al., 2005).

iv) Lupus nephritis patients were assessed clinically with the renal SLE disease activity index [renal SLEDAI] (Chang et al., 2002).

v) Laboratory investigations including:

a) Fasting blood glucose (Burtis et al., 2006). 
b) Renal function tests: blood urea and serum creatinine (Burtis et al., 2006).

c) Serum albumin (Burtis et al., 2006).

d) Complete blood count (CBC) (Bain et al., 2006) and Erythrocyte sedimentation rate (ESR) (Sox and Liang, 1986).

e) C-reactive protein (high sensitivity) (CRP) (Fouda et al., 2012).

f) Complete urine analysis (Burtis et al., 2006), urinary albumin/creatinine ratio in spot urine sample (Cholongitas et al., 2007) and SLICC Renal Activity Score(SLICC RAS) (Petri et al., 2008 ) is calculated.

vi) Ultrasound evaluation of kidneys and renal Doppler with measurement of renal resistance index (RRI) (Kalantarinia, 2009; Rauta et al., 2012).

vii) Renal biopsy: it was done for patients of group I following an informed consent, the coreis examined under light microscopy for staging of LN with estimation of chronicity and activity indices according to the scheme of the International Society of Nephrology/Renal Pathology Society (ISN/RPS)2003classificationof LN (Korbet, 2002; Weening et al., 2004; Zappitelli et al., 2008).

The study protocol was reviewed and approved by the Ethics Committee of the Faculty of Medicine, Alexandria University. After data was collected, it was revised, coded and entered to the statistical software SPSS version 21. Descriptive statistics were used for summarization of data, for quantitative variables, mean and standard deviations (SD) were calculated. Two tailed tests were used and $P$ value of $<0.05$ was considered significant. The Receiver Operating Characteristic (ROC) curve analysis was used to evaluate the predictive value of RRI measured by Doppler Sonography in comparison with disease chronicity score in LN patients (Zweig and Campbell, 1993).

\section{RESULTS}

In this study, group I included 8 males (24.2\%) and 25 females $(75.8 \%)$ with mean age $30.48 \pm 11.94$ years, group II included 2 males (20\%) and 8 females (80\%) with mean age $27.50 \pm 7.65$ years and group III included 5 males (50\%) and 5 females (50\%) with mean age 27.8 \pm 6.12 years with no statistical significant difference between the studied groups as regard age and sex (Table 1). The duration of the disease showed no statistically significant difference between the two groups (Table 2). No statistically significant difference between both groups as regard the presenting symptoms and signs of the studied cases but oedema were present in 7 patients $(21.21 \%)$ of group I and none was found in group II (Table 3). Mean blood pressure (MBP) was within normal range in the studied groups with no statistically significant differences between these groups (Table 4).

The mean value of haemoglobin was statistically significant lower in group I than that of the other 2 groups with no statistically significant difference between group I and group II. White blood cells (WBCs) showed a statistically significant difference between group II and group III but no statistically significant difference between group I and other two groups. The mean value of lymphocytes and platelets had no statistically significant difference between groups (Table 5). The mean value of FBS was within normal in the study groups. It was statistically significant higher in group I than that of group
II and group III with no statistically significant difference between group II and group III. The mean value of serum creatinine was statistically significant higher in group I than that of the other 2 groups with no statistically significant difference between group II and group III. The mean value of blood urea was statistically significant different between the studied groups. The mean value of eGFR was statistically significant lower in group I than group II and group III but no statistically significant difference between group II and III. The mean value of serum albumin in Group I was statistically significant lower than group II and group III with no statistically significant difference between group II and group III. (Table 6)

As regard urine analysis among group I, 5 patients had (nil) proteins in urine and 3 patients had (1 plus) proteins in urine, 7 patients had ( 2 plus) proteins in urine, 15 patients had ( 3 plus) proteins and 3 patients had (4 plus) proteins in urine. Pus cells were ranged between 2 and 100 cells/HPF and RBCs were ranged between 2 and 57 cells/HPF in 9 patients of the LN cases (Table 7). The mean value of urinary albumin creatinine ratio showed statistically significant difference between the studied groups (Table 8)

ESR was statistically significant lower in first and second hour in group III than that of group I and group II with no statistically significant difference between these two groups. The mean value of CRP was statistically significant lower in group III than that of group II with no statistically significant difference between group I and group III or group II (Table 9).

In group I, 32 patients (96.97\%) were positive for antinuclear antibodies while in group II, 9 patients $(88.9 \%)$ were positive for antinuclear antibodies with no statistically significant difference between both groups (Table 10). The mean value of Anti ds-DNA in group I was statistically significant lower in group III than the other two groups but with no statistically significant differences observed between group I and group II. The mean value of C3 was statistically significant lower in group I than that of group II and III but no statistically significant difference between group II and III and the mean value of $\mathrm{C} 4$ was statistically significant lower in group I than that of group III but no statistically significant difference between it in group I and group II or between group II and group III (Table 11).

The mean value of SLEDAl score in group I was statistically significant higher in group I than group II (Table 12). In group I, the mean value of renal SLEDAI was $10.62 \pm 3.14$ and the mean value of SLICCRAS was $9.46 \pm 3.91$ (Table13). As regard renal biopsy, no patient had class I, 3 patients had class II LN, 4 patients had class III LN, 20 patients had class IV LN, 5 patients had class $\mathrm{V} L N$ and only one patient had class VI LN. The mean value of activity index was $7.51 \pm 4.06$ while the mean value of chronicity index was $2.54 \pm 2.07$ (Table 14). 
Table 1. Distribution of the studied groups by demographic data.

\begin{tabular}{|c|c|c|c|c|c|c|c|c|}
\hline \multirow{2}{*}{ Parameter } & \multicolumn{2}{|c|}{ Group I (n = 33) } & \multicolumn{2}{|c|}{ Group II $(n=10)$} & \multicolumn{2}{|c|}{ Group III $(n=10)$} & \multirow{2}{*}{ - Test of sig. } & \multirow[b]{2}{*}{$\mathbf{p}$} \\
\hline & No. & $\%$ & No. & $\%$ & No. & $\%$ & & \\
\hline \multicolumn{9}{|l|}{ Sex } \\
\hline Male & 8 & 24.2 & 2 & 20.0 & 5 & 50.0 & \multirow{2}{*}{$x^{2}=2.928$} & \multirow{2}{*}{${ }^{\mathrm{MC}} \mathrm{p}=0.299$} \\
\hline Female & 25 & 75.8 & 8 & 80.0 & 5 & 50.0 & & \\
\hline \multicolumn{9}{|l|}{ Age (years) } \\
\hline Min - Max & \multirow{2}{*}{\multicolumn{2}{|c|}{$\begin{array}{c}17.0-61.0 \\
30.48 \pm 11.94\end{array}$}} & \multicolumn{2}{|c|}{$19.0-43.0$} & \multicolumn{2}{|c|}{$21.0-40.0$} & & \\
\hline Mean \pm SD & & & \multirow{2}{*}{\multicolumn{2}{|c|}{$\begin{array}{c}27.50 \pm 7.65 \\
24.0\end{array}$}} & \multirow{2}{*}{\multicolumn{2}{|c|}{$\begin{array}{l}27.80 \pm 6.12 \\
26.5\end{array}$}} & $F=0.489$ & $p=0.617$ \\
\hline Median & \multicolumn{2}{|c|}{27.0} & & & & & & \\
\hline
\end{tabular}

Group I: Lupus nephritis group, Group II: Systemic lupus erythematosus group, Group III: Control group.

Table 2. Comparison between patients groups according to duration of the disease.

\begin{tabular}{lcccc}
\hline Duration of the disease (months) & Group I $(\mathbf{n}=\mathbf{3 3})$ & Group II $(\mathbf{n}=\mathbf{1 0})$ & $\mathbf{T}$ & $\mathbf{P}$ \\
\hline Min - Max & $0.25-96$ & $1-11$ & & \\
Mean \pm SD & $12.01 \pm 25.826$ & $4.37 \pm 3.335$ & 0.9255 & 0.360 \\
Median & 3.00 & 3.5 & & \\
\hline
\end{tabular}

Group I: Lupus nephritis group, Group II: Systemic lupus erythematosus group.

Table 3. Presenting symptoms and signs of LN and SLE studied cases.

\begin{tabular}{lccc}
\hline Presenting symptoms and signs & Group I $(\mathbf{n}=\mathbf{3 3})$ & Group II ( $\mathbf{n = 1 0})$ & P \\
\hline Arthritis & $19(57.6 \%)$ & $5(50 \%)$ & 0.727 \\
Alopecia & $14(42.42 \%)$ & $8(80 \%)$ & 0.069 \\
Rash & $14(42.42 \%)$ & $2(20 \%)$ & 0.275 \\
Oral ulcers & $9(27.27 \%)$ & $3(30 \%)$ & 0.999 \\
Fever & $7(21.21 \%)$ & $1(10 \%)$ & 0.656 \\
Oedema & $7(21.21 \%)$ & - & - \\
\hline
\end{tabular}

Group I: Lupus nephritis group, Group II: Systemic lupus erythematosus group. *Fisher exact.

Table 4. Comparison between the different groups according to mean blood pressure.

\begin{tabular}{llll}
\hline MBP $(\mathbf{m m H g})$ & Group I $(\mathbf{n}=\mathbf{3 3})$ & Group II $(\mathbf{n}=\mathbf{1 0})$ & Group III $(\mathbf{n}=\mathbf{1 0})$ \\
\hline Min - Max & $80.00-150.00$ & $90.0-120.0$ & $85.00-110.00$ \\
Mean \pm SD. & $109.48 \pm 18.58$ & $101.87 \pm 10.33$ & $100.00 \pm 7.07$ \\
Median & 105.00 & 97.5 & 100.00 \\
P & $\mathrm{p}=0.124$ & $\mathrm{p}_{1}=0.641$ & $\mathrm{p}_{2}=0.225$ \\
\hline
\end{tabular}

Group I: Lupus nephritis group, Group II: Systemic lupus erythematosus group, Group III: Control group.

$\mathrm{MBP}=$ Mean blood pressure

$\mathrm{P}: \mathrm{P}$ value for comparing between group III with group I p1: P value for comparing between group III and group II p2: P value for comparing between group I and group II.

The mean value of RRI was statistically significant higher in group I than that of group II and group III but no statistically significant difference between group II and group III (Table 15). There was a statistically significant difference between the mean values of creatinine among different lupus nephritis classes (Table 16). In addition serum creatinine and blood urea were statistically significant correlated with renal biopsy chronicity index 
Table 5. Comparison between the different groups according to CBC.

\begin{tabular}{llll}
\hline Parameter & Group I $(\mathbf{n}=\mathbf{3 3})$ & Group II $(\mathbf{n}=\mathbf{1 0})$ & Group III $(\mathbf{n}=\mathbf{1 0})$ \\
\hline HGB $(\mathrm{g} / \mathrm{dl})$ & & & \\
Min - Max & $5.2-13.0$ & $9.30-13.0$ & $11.0-15.0$ \\
Mean \pm SD & $8.8 \pm 1.93$ & $11.08 \pm 2.08$ & $12.9 \pm 1.91$ \\
Median & 8.58 & 10.5 & 13.0 \\
P & $\mathrm{p}=0.001^{*}$ & $\mathrm{p}_{1}=0.0554$ & $\mathrm{p}_{2}=0.0005^{\star}$ \\
WBCs $\left(\mathrm{cell} / \mathrm{mm}^{3}\right)$ & & & \\
Min - Max & $1.70-18.0$ & $4.0-9.50$ & $4.1-7.0$ \\
Mean \pm SD & $6.57 \pm 4.30$ & $6.92 \pm 1.60$ & $5.21 \pm 1.10$ \\
Median & 6.010 & 7.10 & 4.75 \\
P & $\mathrm{p}=0.331$ & $\mathrm{p}_{1}=0.012^{*}$ & $\mathrm{p}_{2}=0.803$ \\
& & & \\
Lymphocytes $\left(\mathrm{cell} / \mathrm{mm}^{3}\right)$ & & & \\
Min - Max & $400.00-5000.00$ & $500.00-5000$ & $1400-3500$ \\
Mean \pm SD & $2.437 \pm 1.34$ & $2.487 \pm 1.52$ & $2.380 \pm 6.62$ \\
Median & 2300.00 & 2550 & 2550 \\
P & $\mathrm{p}=0.989$ & $\mathrm{p}_{1}=0.839$ & $\mathrm{p}_{2}=0.921$ \\
& & & \\
Platelets $\left(\mathrm{cell} / \mathrm{mm}^{3}\right)$ & & & $145.0-210.0$ \\
Min - Max & $16.0-389$. & $5.0-518.0$ & $174.7 \pm 212.91$ \\
Mean \pm SD & $205.6 \pm 104.65$ & $203.5 \pm 147.0$ & 173.0 \\
Median & 188.50 & 208.0 & $\mathrm{p}_{2}=0.960$ \\
P & $\mathrm{p}=0.532$ & $\mathrm{p}_{1}=0.728$ & \\
\hline
\end{tabular}

Group I: Lupus nephritis group, Group II: Systemic lupus erythematosus group, Group III: Control group. $\mathrm{CBC}=$ Complete blood count $\mathrm{HGB}=$ Haemoglobin WBCs $=$ White blood cells

p: P value for comparing between group I with group III

$\mathrm{p}_{1}$ : $\mathrm{P}$ value for comparing between group II and group III

$\mathrm{p}_{2}$ : $P$ value for comparing between group I and group II.

Table 6. Comparison between the different groups according to some laboratory parameters.

\begin{tabular}{llll}
\hline Parameter & Group I $(\mathbf{n}=\mathbf{3 3})$ & Group II $(\mathbf{n}=\mathbf{1 0})$ & Group III $(\mathbf{n}=\mathbf{1 0})$ \\
\hline FBS $(\mathrm{mg} / \mathrm{dl})$ & & & \\
Min - Max & $75-99$ & $79-98$ & $78-89$ \\
Mean \pm SD & $92.8 \pm 5.45$ & $85.12 \pm 6.91$ & $82.3 \pm 3.68$ \\
Median & 93 & 83.3 & 81.5 \\
P & $\mathrm{p}=0.0001^{*}$ & $\mathrm{p}_{1}=0.1004$ & $\mathrm{p}_{2}=0.0007^{\star}$ \\
& & \\
Serum creatinine (mg/dl) & & & \\
Min - Max & $0.4-9.80$ & $0.4-1.0$ & $0.40-0.90$ \\
Mean \pm SD & $2.02 \pm 2.03$ & $0.71 \pm 0.18$ & $0.68 \pm 0.147$ \\
Median & 1.30 & 0.75 & 0.70 \\
P & $\mathrm{p}=0.045^{*}$ & $\mathrm{p}_{1}=0.687$ & $\mathrm{p}_{2}=0.049^{*}$ \\
& & & \\
Blood urea (mg/dl) & & & \\
Min - Max & $20.00-180.00$ & $16.00-47.00 \mathrm{~g}$ & $14.00-27.00$ \\
Mean \pm SD & $66.58 \pm 46.58$ & $32.12 \pm 10.37$ & $20.90 \pm 3.84$ \\
Median & 49.00 & 29.50 & 20.50 \\
$\mathrm{P}$ & $\mathrm{P}=0.0038^{*}$ & $\mathrm{p}_{1}=0.0049^{*}$ & $\mathrm{p}_{2}=0.0264^{*}$ \\
\hline
\end{tabular}


Table 6. Continues.

\begin{tabular}{llll}
\hline eGFR $(\mathrm{ml} / \mathrm{min})$ & & & \\
Min - Max & $5.0-120.0$ & $68.0-120.0$ & $100.0-117.0$ \\
Mean \pm SD & $55.85 \pm 36.676$ & $101.25 \pm 19.717$ & $107.40 \pm 5.316$ \\
Median & 45.5000 & 105.5000 & 107.50 \\
P & $\mathrm{p}=0.0001^{*}$ & $\mathrm{p}_{1}=0.3535$ & $\mathrm{p}_{2}=0.0006^{*}$ \\
& & & \\
Serum albumin (g/dl) & & & \\
Min - Max & $1.10-4.1$ & $2.40-4.50$ & $2.0-4.0$ \\
Mean \pm SD & $2.46 \pm 0.67$ & $3.68 \pm 0.762$ & $3.68 \pm 0.617$ \\
Median & 2.50 & 4.00 & 3.8 \\
$\mathrm{P}$ & $\mathrm{p}=0.001^{*}$ & $\mathrm{p}_{1}=0.999$ & $\mathrm{p}_{2}=0.0001^{*}$ \\
\hline
\end{tabular}

Group I: Lupus nephritis group, Group II: Systemic lupus erythematosus group, Group III: Control group

FBS $=$ Fasting blood sugar.

eGFR = estimated glomerularfiltration rate

p: $P$ value for comparing between group III with group I

$\mathrm{p}_{1}$ : $\mathrm{P}$ value for comparing between group III and group II

$\mathrm{p}_{2}$ : $\mathrm{P}$ value for comparing between group I and group II.

Table 7. Complete urine analysis in lupus nephritis patients.

\begin{tabular}{|c|c|c|}
\hline \multirow{2}{*}{ Variable } & \multicolumn{2}{|c|}{ Group I (n =33) } \\
\hline & No. & $\%$ \\
\hline \multicolumn{3}{|l|}{ Proteins } \\
\hline Nil & 5 & 15.2 \\
\hline 1 plus & 3 & 9.1 \\
\hline 2 plus & 7 & 21.2 \\
\hline 3 plus & 15 & 45.4 \\
\hline 4 plus & 3 & 9.1 \\
\hline Casts & 9 & 22.27 \\
\hline \multicolumn{3}{|l|}{ Pus cells } \\
\hline Min - Max & \multicolumn{2}{|c|}{$2.0-100$} \\
\hline Mean \pm SD & \multicolumn{2}{|c|}{$11.08 \pm 18.43$} \\
\hline Median & \multicolumn{2}{|c|}{7.0} \\
\hline \multicolumn{3}{|l|}{ Red cells } \\
\hline Min - Max & \multicolumn{2}{|c|}{$2-57$} \\
\hline Mean \pm SD & \multicolumn{2}{|c|}{$17.80 \pm 16.30$} \\
\hline Median & \multicolumn{2}{|c|}{10.0} \\
\hline
\end{tabular}

Group I: Lupus nephritis group.

(Table 17). Out of $33 \mathrm{LN}$ cases, 6 patients had RRI of 0.7 and above giving a percentage of $18.18 \%$.

The correlation of some demographic, clinical and laboratory variables with RRI showed that age, MBP, blood urea, serum creatinine, HB, urinary AC ratio, FBS, eGFR, ESR2, serum C3 and chronicity index in renal biopsy were statistically significant correlated with the RRI (Table 18). Multiple regression analysis showed that no variable was a significant predictor for Doppler-Based
RRI (Table 19).

The main demographic and clinical features of LN patients grouped with respect to the RRI value. Lupus nephritis patients with $R R I \geq 0.7(n=6)$ had statistically significant higher mean age, mean serum creatinine, blood urea level and higher chronicity index compared with patients with RRI in the normal range (Table 20). No statistically significant difference was observed between renal biopsy classes and RRI greater than 0.7 (Table 21).

In Figure 1, it appears that the Area under the ROC curve (AUC) was 0.769 (95\% confidence interval 0.587 to $0.950, P=0.019$ ). The AUC was statistically significant different from 0.5 (null hypothesis area) and therefore there is evidence that Doppler-Based RRI has an ability to distinguish between the positive and negative groups.

\section{DISCUSSION}

Lupus nephritis (LN) affects up to $60 \%$ of patients with SLE, either as the initial manifestation or during the course of the disease (Boumpas et al., 1995). Moreover, $\mathrm{LN}$ has a negative impact on survival of patients with lupus (Cervera et al., 2003). It is extremely heterogenous both clinically and pathologically, flares and remissions of diseases are usually associated with therapy (Moc and Lau, 2003). Valesini and Conti (2011) considered that prognostic factors associated with poor outcome include: non-white ethnicity, male gender, uncontrolled hypertension, and high activity and chronicity indices on kidney biopsy. Accordingly, it is mandatory to identify markers of LN severity able to guide clinicians toward the adequate therapeutic option (Mok, 2010).

The most important survey of $L N$ is histological assessment by renal biopsy. Clinicians usually select appropriate therapy according to the results of renal 
Table 8. Comparison between the different groups according to urinary albumin creatinine ratio.

\begin{tabular}{llll}
\hline Urinary AC ratio $(\mathbf{m g} / \mathbf{g})$ & Group I $(\mathbf{n}=\mathbf{3 3})$ & Group II $(\mathbf{n}=\mathbf{1 0})$ & Group III $(\mathbf{n}=\mathbf{1 0})$ \\
\hline Min - Max & $788.00-17000.00$ & $120.00-143.00$ & $87.00-99.00$ \\
Mean \pm SD & $3578.42 \pm 3546.13$ & $132.62 \pm 9.650$ & $91.80 \pm 4.13$ \\
Median & 2661.50 & 131.0 & 92.00 \\
$\mathbf{P}$ & $\mathrm{p}=0.0038^{*}$ & $\mathrm{p}_{1}=0.0001^{*}$ & $\mathrm{p}_{2}=0.004^{*}$ \\
\hline
\end{tabular}

Group I: Lupus nephritis group, Group II: Systemic lupus erythematosus group, Group III: Control group Urinary $A C$ ratio= Urinary albumin creatinine ratio

$\mathrm{p}$ : $\mathrm{P}$ value for comparing between group III with group I

$\mathrm{p}_{1}$ : $\mathrm{P}$ value for comparing between group III and group II

$\mathrm{p}_{2}$ : $\mathrm{P}$ value for comparing between group I and group II.

Table 9. Comparison between the different groups according to ESR and CRP.

\begin{tabular}{llll}
\hline Variable & Group I $(\mathbf{n}=\mathbf{3 3})$ & Group II $(\mathbf{n}=\mathbf{1 0})$ & Group III $(\mathbf{n}=\mathbf{1 0})$ \\
\hline ESR1 $(\mathrm{mm} /$ hour $)$ & & & \\
Min - Max & $10-137$ & $43-120$ & $15-24$ \\
Mean \pm SD & $55.27 \pm 35.82$ & $79.83 \pm 32.10$ & $20.08 \pm 2.83$ \\
Median & 45.0 & 77.5 & 20.5 \\
P & $P=0.0037^{*}$ & $p_{1}=0.0001^{*}$ & $p_{2}=0.059$ \\
& & \\
ESR2 (mm/hour) & & & \\
Min - Max & $20-150$ & $80-160$ & $18-29$ \\
Mean \pm SD & $87.42 \pm 42.09$ & $114.0 \pm 31.15$ & $25.90 \pm 3.63$ \\
Median & 80 & 105 & 29 \\
$P$ & $p=0.0001^{*}$ & $p_{1}=0.0001^{*}$ & $p_{2}=0.0725$ \\
& & & \\
CRP (mg/L) & & & \\
Min - Max & $2-131$ & $3-29$ & $1-5$ \\
Mean \pm SD & $18.57 \pm 28.808$ & $16.63 \pm 11.045$ & $2.6 \pm 1.430$ \\
Median & 5.00 & 21.50 & 2.5 \\
$P$ & $\mathrm{p}=0.0898$ & $\mathrm{p}_{1}=0.0009^{*}$ & $\mathrm{p}_{2}=0.8371$ \\
\hline
\end{tabular}

Group I: Lupus nephritis group, Group II: Systemic lupus erythematosus group, Group III: Control group $\mathrm{ESR}=$ Erythrocyte sedimentation rate

ESR1 = Erythrocyte sedimentation rate in first hour

$\mathrm{ESR} 2=$ Erythrocyte sedimentation rate in second hour

$\mathrm{CRP}=\mathrm{C}$-reactive protein

$\mathrm{p}: \mathrm{P}$ value for comparing between group III with group I

$\mathrm{p}_{1}$ : $\mathrm{P}$ value for comparing between group III and group II

$\mathrm{p}_{2}$ : $\mathrm{P}$ value for comparing between group I and group II.

Table 10. Comparison between patients groups according to antinuclear antibody.

\begin{tabular}{lccc}
\hline \multirow{2}{*}{ Group } & \multicolumn{2}{c}{ Antinuclear antibody } & \multirow{2}{*}{ Total (\%) } \\
\cline { 2 - 3 } & Positive (\%) & Negative (\%) & \\
\hline Group I & $32(96.97)$ & $1(3.03)$ & $33(100.0)$ \\
Group II & $9(88.9)$ & $1(11.1)$ & $10(100.0)$ \\
\hline
\end{tabular}

Group I: Lupus nephritis group, Group II: Systemic lupus erythematosus group. Fisher exact $\mathrm{P}=0.422$.

biopsy. However, since renal biopsy is an invasive procedure, a non-invasive alternative would have clinical benefits (Gao et al., 2013).

Conventional ultrasound does not provide any 
Table 11. Comparison between the different groups according to immunological markers.

\begin{tabular}{llll}
\hline Variable & Group I $(\mathbf{n}=\mathbf{3 3})$ & Group II $(\mathbf{n}=\mathbf{1 0})$ & Group III $(\mathbf{n}=\mathbf{1 0})$ \\
\hline Anti-ds DNA (IU/ml) & & & \\
Min - Max & $24-1200$ & $30-980$ & $11-29$ \\
Mean \pm SD & $217.38 \pm 279.320$ & $257.13 \pm 342.24$ & $17.60 \pm 7.214$ \\
Median & 78.00 & 118 & 14.5 \\
P & $\mathrm{p}=0.0311$ & $\mathrm{p}_{1}=0.0045$ & $\mathrm{p}_{2}=0.736$ \\
& & & \\
C3 (mg/dl) & & & $100-170$ \\
Min - Max & $15-107$ & $20-140$ & $121.5 \pm 23.34$ \\
Mean \pm SD & $57.2 \pm 27.62$ & $98.13 \pm 36.54$ & 110 \\
Median & 55.00 & 105.5 & $\mathrm{p}_{2}=0.0004^{*}$ \\
P & $\mathrm{p}=0.0001^{*}$ & $\mathrm{p}_{1}=0.1004$ & \\
& & & $11-28$ \\
C4 (mg/dl) & & $2-35$ & $20.50 \pm 5.25$ \\
Min - Max & $2-41$ & $15.7 \pm 10.76$ & 20.5 \\
Mean \pm SD & $12.93 \pm 9.81$ & 4.00 & $\mathrm{p}_{2}=0.4485$ \\
Median & 9.0 & $\mathrm{p}_{1}=0.2211$ & \\
P & $\mathrm{p}=0.0249^{*}$ &
\end{tabular}

Group I: Lupus nephritis group, Group II: Systemic lupus erythematosus group, Group III: Control group C3= Complement 3

$\mathrm{C} 4=$ Complement 4

p: P value for comparing between group III with group I

$\mathrm{p}_{1}$ : P value for comparing between group III and group II

$\mathrm{p}_{2}$ : $\mathrm{P}$ value for comparing between group I and group II.

Table 12. Comparison between patients groups according to SLEDAI score.

\begin{tabular}{lllll}
\hline SLEDAI score & Group I $(\mathbf{n}=\mathbf{3 3})$ & Group II $(\mathbf{n}=\mathbf{1 0})$ & $\mathbf{t}$ & $\mathbf{P}$ \\
\hline Min - Max & $4-30$ & $6-14$ & & \\
Mean \pm SD & $21.32 \pm 6.28$ & $10.75 \pm 3.19$ & 5.096 & $0.0001^{*}$ \\
Median & 24 & 11 & & \\
\hline
\end{tabular}

Group I: Lupus nephritis group, Group II: Systemic lupus erythematosus group. SLEDAI = Systemic lupus erythematosus disease activity index.

Table 13. Renal SLEDAI and SLICCRAS scores in lupus nephritis patients.

\begin{tabular}{ll}
\hline Variable & Group I $(\mathbf{n}=\mathbf{3 3})$ \\
\hline Renal SLEDAI & \\
Min - Max & $4.0-16.0$ \\
Mean \pm SD & $10.62 \pm 3.14$ \\
Median & 12.0 \\
& \\
SLICCRAS & \\
Min - Max & $3.0-15.0$ \\
Mean \pm SD & $9.46 \pm 3.91$ \\
Median & 9.0 \\
\hline
\end{tabular}

Group I: Lupus nephritis group

Renal SLEDAI = Renal systemic lupus erythematosus disease activity index

SLICCRAS = Systemic lupus international collaborating clinics renal activity score. additional predictive information over the traditional serological and biopsy parameters (Ozbek et al., 1995). Doppler analysis allows acquisition of information on renal macro-abnormalities and changes in renal blood flow. Renal resistive index (RRI) is commonly used as an index of intra-renal arterial resistance, and its use is proposed in the differential diagnosis of several nephropathies (Parolini et al., 2009; Rosato et al., 2012; Krumme, 2006).

The goal of our study was to evaluate the predictive value of RRI by Doppler US in LN as compared to disease activity scores, serological and biopsy parameters.

Group I included 25 females and 8 males with mean age $30.48 \pm 11.94$ years, and group II included 8 females and 2 males with a mean age $27.80 \pm 6.12$ years. Most reported data demonstrated that SLE occur in up to 10 times more common in women than men, and typically 
Table 14. Distribution of the studied cases according to renal biopsy in lupus nephritis.

\begin{tabular}{|c|c|c|}
\hline Variable & Group I $(n=33)$ & $\%$ \\
\hline \multicolumn{3}{|l|}{ Class } \\
\hline II Mesangial proliferative lupus nephritis & 3 & 9.09 \\
\hline III Focal lupus nephritis & 4 & 12.12 \\
\hline IV Diffuse lupus nephritis & 20 & 60.60 \\
\hline V Membranous lupus nephritis & 5 & 15.15 \\
\hline VI Advanced sclerotic lupus nephritis & 1 & 3.03 \\
\hline \multicolumn{3}{|l|}{ Activity index } \\
\hline Min - Max & \multicolumn{2}{|c|}{$1.0-15.0$} \\
\hline Mean \pm SD & \multicolumn{2}{|c|}{$7.51 \pm 4.06$} \\
\hline Median & \multicolumn{2}{|c|}{8.0} \\
\hline \multicolumn{3}{|l|}{ Chronicity index } \\
\hline Min - Max & \multicolumn{2}{|c|}{$0-8.0$} \\
\hline Mean \pm SD & \multicolumn{2}{|c|}{$2.54 \pm 2.07$} \\
\hline Median & \multicolumn{2}{|c|}{2.0} \\
\hline
\end{tabular}

Group I = Lupus nephritis group.

Table 15. Comparison between the different groups according to renal resistive index.

\begin{tabular}{llll}
\hline RRI & Group I $(\mathbf{n}=\mathbf{3 3})$ & Group II $(\mathbf{n}=\mathbf{1 0})$ & Group III $(\mathbf{n}=\mathbf{1 0})$ \\
\hline Min - Max & $0.56-0.74$ & $0.52-0.61$ & $0.54-0.57$ \\
Mean \pm SD & $0.65 \pm 0.05$ & $0.58 \pm 0.03$ & $0.55 \pm 0.10$ \\
Median & 0.65 & 0.58 & 0.55 \\
$\mathrm{P}$ & $\mathrm{p}=0.0001^{*}$ & $\mathrm{p}_{1}=0.3755$ & $\mathrm{p}_{2}=0.0001^{*}$ \\
\hline
\end{tabular}

Group I: Lupus nephritis group, Group II: Systemic lupus erythematosus group, Group III: Control group

$\mathrm{RRI}=$ Renal resistive index

$P: P$ value for comparing between group III with group I

$P 1$ : $P$ value for comparing between group III and group II

P2: P value for comparing between group I and group II.

Table 16. Distribution of renal biopsy classes by creatinine value in lupus nephritis patients.

\begin{tabular}{lcc}
\hline Class $(\mathbf{n}=\mathbf{3 3})$ & Mean & SD \\
\hline II Mesangial proliferative lupus nephritis & 0.7000 & 0.26458 \\
III Focal lupus nephritis & 1.9000 & 1.31149 \\
IV Diffuse lupus nephritis & 2.3409 & 2.58174 \\
V Membranous lupus nephritis & 1.9750 & 2.22017 \\
VI Advanced sclerotic lupus nephritis & 9.8000 & - \\
Total & 2.3333 & 2.64086 \\
\hline
\end{tabular}

$F=2.866, P=0.042$.

Table 17. Correlation of both creatinine and blood urea values with renal biopsy chronicity index.

\begin{tabular}{lll}
\hline Variable & & Renal biopsy chronicity index \\
\hline Serum creatinine $(\mathrm{mg} / \mathrm{dl})$ & Pearson correlation & $0.576^{\star}$ \\
& P & 0.000 \\
& & \\
Blood urea $(\mathrm{mg} / \mathrm{dl})$ & Pearson correlation & $0.449^{\star}$ \\
& P & 0.009 \\
\hline
\end{tabular}


Table 18. Correlation of some demographic, clinical and laboratory variables with RRI.

\begin{tabular}{|c|c|c|}
\hline Variable & & $\mathbf{R R I}$ \\
\hline \multirow{2}{*}{ Age (years) } & Pearson Correlation & $0.314^{*}$ \\
\hline & $\mathrm{P}$ & 0.032 \\
\hline \multirow{2}{*}{$\mathrm{HB}(\mathrm{g} / \mathrm{dl})$} & Pearson Correlation & $-0.419^{*}$ \\
\hline & $P$ & 0.002 \\
\hline \multirow{2}{*}{ FBS (mg/dl) } & Pearson Correlation & $0.365^{*}$ \\
\hline & $P$ & 0.007 \\
\hline \multirow{2}{*}{ MBP $(\mathrm{mmHg})$} & Pearson Correlation & $0.482^{*}$ \\
\hline & $\mathrm{P}$ & 0.000 \\
\hline \multirow{2}{*}{ eGFR (ml/min) } & Pearson Correlation & $-0.462^{*}$ \\
\hline & $P$ & 0.013 \\
\hline \multirow{2}{*}{ Creatinine (mg/dl) } & Pearson Correlation & $0.709^{*}$ \\
\hline & $P$ & 0.000 \\
\hline \multirow{2}{*}{ Blood urea (mg/dl) } & Pearson Correlation & $0.647^{*}$ \\
\hline & $P$ & 0.000 \\
\hline \multirow{2}{*}{ Serum albumin $(\mathrm{g} / \mathrm{dl})$} & Pearson Correlation & -0.057 \\
\hline & $\mathrm{P}$ & 0.693 \\
\hline \multirow{2}{*}{ Urinary AC Ratio (mg/g) } & Pearson Correlation & $0.315^{*}$ \\
\hline & $\mathrm{P}$ & 0.024 \\
\hline \multirow{2}{*}{ ESR2 (mm/hour) } & Pearson Correlation & $0.301^{*}$ \\
\hline & $\mathrm{P}$ & 0.034 \\
\hline \multirow{2}{*}{ Anti ds DNA (IU/L) } & Pearson Correlation & 0.123 \\
\hline & $P$ & 0.381 \\
\hline \multirow{2}{*}{ Serum C3 (mg/dl) } & Pearson Correlation & $-0.438^{*}$ \\
\hline & $\mathrm{P}$ & 0.001 \\
\hline \multirow{2}{*}{ Serum C4 (mg/dl) } & Pearson Correlation & -0.175 \\
\hline & $\mathrm{P}$ & 0.209 \\
\hline \multirow{2}{*}{ Activity index } & Pearson Correlation & 0.069 \\
\hline & $P$ & 0.704 \\
\hline \multirow{2}{*}{ Chronicity index } & Pearson Correlation & ${ }^{*} 0.443$ \\
\hline & $P$ & 0.010 \\
\hline \multirow{2}{*}{ Renal biopsy classes } & Spearman correlation & 0.243 \\
\hline & $P$ & 0.174 \\
\hline \multirow{2}{*}{ SLEDAI } & Pearson Correlation & 0.120 \\
\hline & $\mathrm{P}$ & 0.450 \\
\hline \multirow{2}{*}{ SLICCRAS } & Pearson Correlation & -0.100 \\
\hline & $\mathrm{P}$ & 0.585 \\
\hline
\end{tabular}


Table 18. Continues.

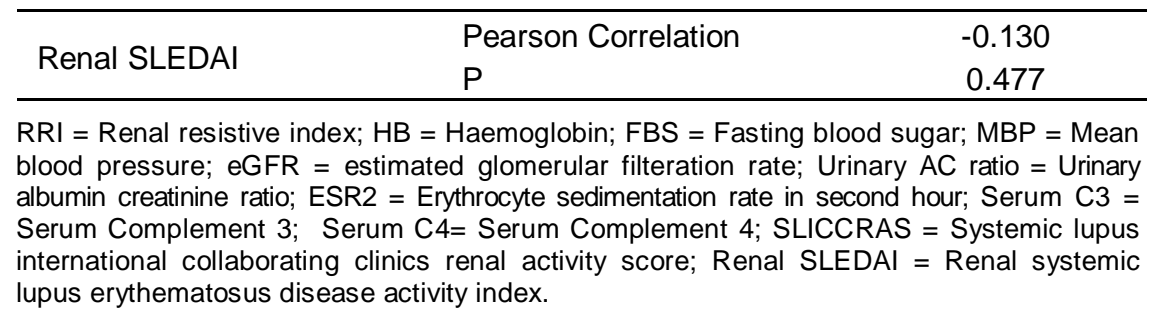

Table 19. Multiple regression analysis of Doppler-Based Renal Resistive index.

\begin{tabular}{|c|c|c|c|c|c|}
\hline \multirow[t]{2}{*}{ Variables } & \multirow{2}{*}{$\begin{array}{c}\begin{array}{c}\text { Standardized } \\
\text { coefficients }\end{array} \\
\text { Beta } \\
\end{array}$} & \multirow[t]{2}{*}{$\mathbf{t}$} & \multirow[t]{2}{*}{ Sig. } & \multicolumn{2}{|c|}{$95.0 \%$ confidence interval for $B$} \\
\hline & & & & Lower bound & Upper bound \\
\hline (Constant) & & 2.338 & .037 & 0.054 & 1.519 \\
\hline Age & -.181 & -.684 & .507 & -.003 & 0.002 \\
\hline $\mathrm{HB}$ & .134 & .535 & .602 & -.010 & 0.016 \\
\hline FBS & -.211 & -.750 & .468 & -.009 & 0.005 \\
\hline ESR & .039 & .145 & .887 & -.001 & 0.001 \\
\hline MBP & .157 & .460 & .654 & -.001 & 0.002 \\
\hline eGFR & .054 & .122 & .834 & -.001 & 0.001 \\
\hline Creatinine & .632 & 1.253 & .234 & -.017 & 0.061 \\
\hline Blood urea & -.086 & -.223 & .827 & -.001 & 0.001 \\
\hline Urinary $A C$ ratio & -.192 & -.698 & .499 & .000 & 0.000 \\
\hline Serum C3 & -.104 & -.354 & .730 & -.001 & 0.001 \\
\hline Renal biopsy chronicity index & .140 & .495 & .630 & -.013 & 0.020 \\
\hline
\end{tabular}

$\mathrm{Hb}=$ Haemoglobin; FBS = Fasting blood sugar; ESR= Erythrocyte sedimentation rate; MBP = Mean blood pressure; eGFR = Estimated glomerular filtration rate; Urinary AC ratio = Urinary albumin creatinine ratio; $\mathrm{C} 3=$ Complement 3.

has a predilection for women in their childbearing years (Uramoto et al., 1999).

The duration of the disease showed no statistically significant difference between the two groups (Ballow et al., 1996) stated that LN occurs early in the disease mostly during the $1^{\text {st }}$ year, and it is uncommon to have the original onset of renal disease more than 10 years after the appearance of SLE.

The mean value of $\mathrm{Hb}$ was statistically significant lower in patients with group I than those of group II. This may be attributed to greater activity or prior use of aggressive immune suppression in group I. (Satirapoj et al., 2007) reported that $\mathrm{Hb}<10 \mathrm{~m}$ is a major determinant of renal involvement in SLE patients. The mean value of WBCs count in group II was statistically significant higher than that of group III but the mean value of lymphocytes and platelets showed no statistically significant between the studied groups. However (Pitashny et al., 2007 and ElShehaby et al., 2011) have found insignificant difference between groups regarding WBCs as well as platelets. This may reflect higher hematological activity in group I evidenced by both lower mean $\mathrm{Hb}$ and WBCs, also there were some patients in group I giving history of aggressive myelotoxic therapy prior to involving in the study.
That mean value of serum creatinine was statistically significant higher in group I than that of the other 2 groups with no statistically significant difference between group II and group III. The mean value of blood urea was statistically significant different between the studied groups. The mean value of eGFR was statistically significant lower in group I than that of group II and group III. This may be attributed to that more than $70 \%$ of group I belong to classes III and IV in renal biopsy and usually patients in these classes have impaired renal functions (Najafi et al., 2001).

The mean value of serum albumin in group I was statistically significant lower than in group II and group III. This might be explained by the fact that Group I with LN had significantly higher mean value of urinary albumin loss. These findings matched with those in Yip et al. (2010), where in 1078 patients with LN and SLE without nephritis, low serum albumin level is significantly associated with LN activity and higher SLEDAl scores. The mean value of UACR was statistically significant higher in group I than group II and III. The use of UACR was also used by Birmingham et al. (2008) were it was found that it is a better forecaster for proteinuric renal flare allowing early declaration of renal flare especially in 
Table 20. Demographic and clinical features of lupus nephritis patients grouped according to RRI value.

\begin{tabular}{|c|c|c|c|}
\hline Variable & $\mathrm{RI}<0.7(\mathrm{~N}=27)$ & $\mathrm{RI}>0.7(\mathrm{~N}=6)$ & $p$ \\
\hline Males/females & $5 / 22$ & $3 / 3$ & 0.104 \\
\hline Age(years) & $28.12 \pm 9.465$ & $41.80 \pm 17.020$ & $0.017^{*}$ \\
\hline Disease duration (months) & $14.90 \pm 29.129$ & $2.41 \pm 1.941$ & 0.487 \\
\hline $\mathrm{MBP}(\mathrm{mmHg})$ & $108.12 \pm 18.285$ & $116.00 \pm 20.736$ & 0.398 \\
\hline \multicolumn{4}{|l|}{ Renal function } \\
\hline Serum creatinine (mg/dl) & $1.46 \pm 0.914$ & $5.32 \pm 3.016$ & $0.000^{*}$ \\
\hline Blood urea(mg/dL) & $57.50 \pm 37.463$ & $110.20 \pm 65.155$ & $0.018^{*}$ \\
\hline eGFR (mL/min) & $60.65 \pm 34.427$ & $33.80 \pm 42.669$ & 0.141 \\
\hline Urine protein(g/24h) & $1.62 \pm 0.868$ & $3.90 \pm 3.726$ & 0.241 \\
\hline \multicolumn{4}{|l|}{ Inflammatory markers } \\
\hline ESR & $59.54 \pm 35.671$ & $34.80 \pm 32.151$ & 0.164 \\
\hline CRP & $19.79 \pm 31.032$ & $12.70 \pm 14.848$ & 0.625 \\
\hline \multicolumn{4}{|l|}{ Immunologic parameters } \\
\hline Antinuclear Antibody & $26(96.3 \%)$ & $6(100 \%)$ & 0.632 \\
\hline Anti ds DNA & $240.08 \pm 301.583$ & $99.60 \pm 78.951$ & 0.317 \\
\hline Serum C3 & $55.57 \pm 27.924$ & $65.00 \pm 27.650$ & 0.497 \\
\hline Serum C4 & $12.79 \pm 9.738$ & $13.62 \pm 11.324$ & 0.867 \\
\hline SLEDAI & $21.95 \pm 5.520$ & $17.50 \pm 9.983$ & 0.195 \\
\hline SLICCRAS & $10.20 \pm 4.010$ & $6.25 \pm 2.061$ & 0.067 \\
\hline Renal SLEDAI & $10.66 \pm 3.472$ & $11.00 \pm 2.00$ & 0.855 \\
\hline \multicolumn{4}{|l|}{ Histologic class } \\
\hline Class II & $3(11.11 \%)$ & - & \\
\hline Class III & $3(11.11 \%)$ & $1(16.67 \%)$ & 0.202 \\
\hline Class IV & $17(62.96 \%)$ & $3(50 \%)$ & \\
\hline Class V & $4(14.82 \%)$ & $1(16.67 \%)$ & \\
\hline Class V1 & - & $1(16.67 \%)$ & \\
\hline Activity Index (range: $0-24$ ) & $7.87 \pm 3.904$ & $6.40 \pm 2.966$ & 0.434 \\
\hline Chronicity Index (range: $0-12$ ) & $2.16 \pm 1.522$ & $4.4000 \pm 2.607$ & $0.014^{*}$ \\
\hline
\end{tabular}

$\mathrm{RRI}=$ renal resistive index; MBP = Mean blood pressure; eGFR = estimated glomerular filtration rate; ESR = Erythrocyte sedimentation rate; CRP = C- reactive protein; Serum C3 = Serum complement 3; Serum C4 = Serum complement 4; SLICCRAS = Systemic lupus international collaborating clinics renal activity score; SLEDAI = Systemic lupus erythematosus disease activity index.

normal range proteinuria avoiding greater nephrotoxicity of proteinuria when using higher threshold protein creatinine ratio.

The mean value of ESR in group III was statistically significant lower than that of group I and group II in first and second hour, these findings matched with those of Stojan et al. (2013) where ESR is associated with disease activity in SLE and with organ-specific activity including serositis, rash, joint, renal and hematological affection. Also CRP was statistically significantly lower in group III than that of group II, this may be explained by that many patients in group II were known to suffer from infections (Mok et al., 2013) showed that CRP is detectable in $77 \%$ of lupus patients with active disease and that CRP level correlate significantly with lupus disease activity, especially that involving the musculoskeletal system, hematological system, and serositis as in most of our patients in group II.

The majority (96.6\%) of LN patients were positive for ANAs and (88.9\%) among SLE patients with no statistical significant difference between both groups. This agrees with the data reporting ANAs are a highly sensitive screen for SLE, being found in more than $90 \%$ of untreated patients, but they are not specific for SLE and occur in many other rheumatologic and nonrheumatologic conditions (D'Agati, 1998). The mean value of Anti ds-DNA in group I and group II was statistically significant higher than that of group III with no significant 
Table 21. Distribution of renal biopsy classes by renal resistive index.

\begin{tabular}{|c|c|c|c|c|}
\hline \multirow{2}{*}{\multicolumn{2}{|c|}{ Renal biopsy classes }} & \multicolumn{2}{|c|}{$\mathbf{R I}$} & \multirow{2}{*}{ Total } \\
\hline & & $<0.7$ & $0.7+$ & \\
\hline \multirow{2}{*}{ II } & No. & 3 & - & 3 \\
\hline & $\%$ & $100.0 \%$ & - & $100.0 \%$ \\
\hline \multirow{2}{*}{ III } & No. & 3 & 1 & 4 \\
\hline & $\%$ & $75.0 \%$ & 25.5 & $100.0 \%$ \\
\hline \multirow{2}{*}{ IV } & No. & 17 & 3 & 20 \\
\hline & $\%$ & $85.0 \%$ & $15.0 \%$ & $100.0 \%$ \\
\hline \multirow{2}{*}{ V } & No. & 4 & 1 & 5 \\
\hline & $\%$ & $80.0 \%$ & $20.0 \%$ & $100.0 \%$ \\
\hline \multirow{2}{*}{$\mathrm{VI}$} & No. & - & 1 & 1 \\
\hline & $\%$ & - & $100.0 \%$ & $100.0 \%$ \\
\hline
\end{tabular}

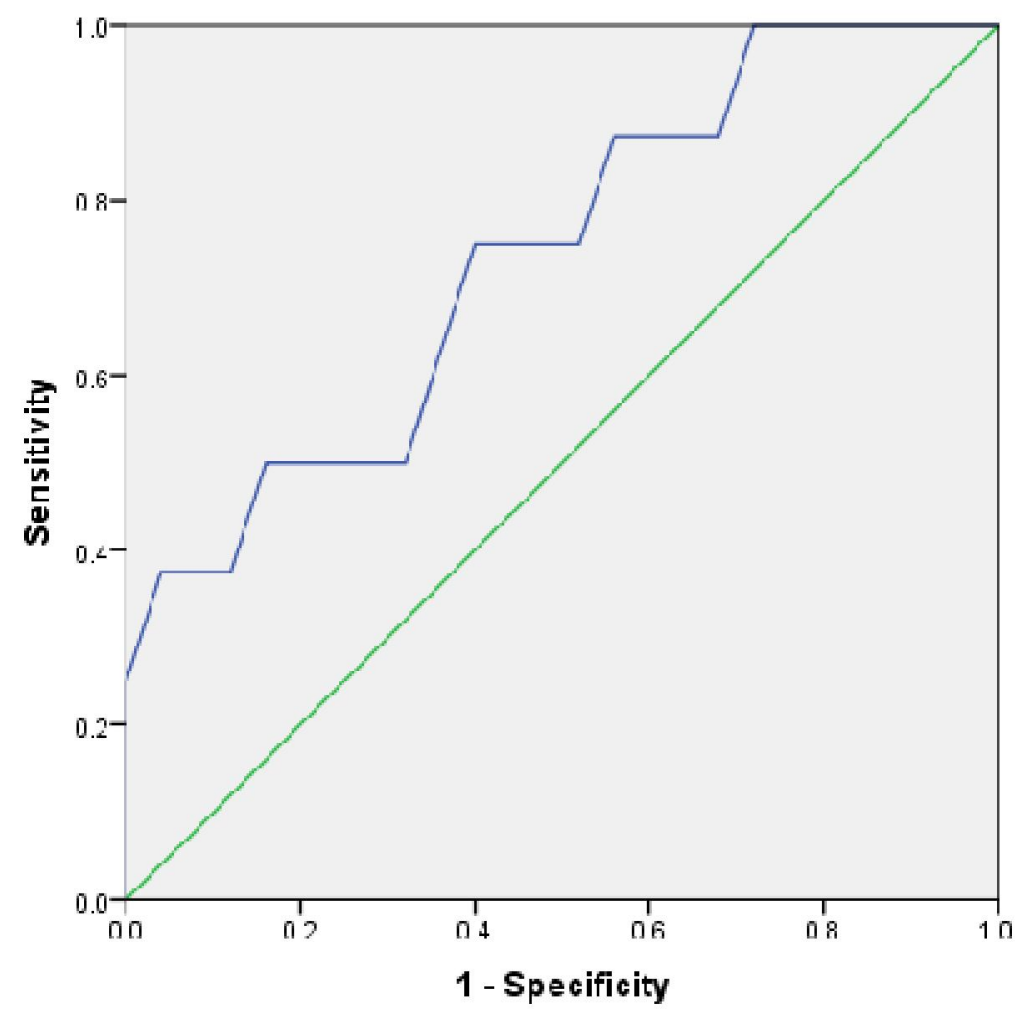

Figure 1. ROC curve of Doppler-Based RRI as a predictor of renal biopsy.

differences observed between group I and group II. AntidsDNA IgG antibodies of high avidity that fix complement have correlated best with the presence of renal disease (Rahman and Isenberg, 2008). Also, Farid et al. (2013) found that anti-ds-DNA was higher in the LN group compared with the non-LN group, showing that patients with active LN often have raised levels of anti-ds DNA antibodies. In addition their titers correlate well with clinical activity as was studied by Linnik et al. (2005).

The mean value of C3 was statistically significant lower in group I than that of group II and group III with no statistical significant difference between group II and group III. While for $\mathrm{C} 4$; it was statistically significant lower in group I than group III while it did not differ significantly 
between group I and group II nor group II and group III. Similarly (Narayanan et al., 2010) has found marked reduction in C3 and C4 levels in LN patients than in SLE patients without renal affection.

The mean value of the SLEDAI score in group I was statistically significant higher than that of group II. This matched with findings of (Zabaleta-Lans et al., 2006). Renal SLEDAI was ranged from 4 to 16 and SLICCRAS was ranged from 3 to 15 . This denoting that most of the patients enrolled in the study are active proliferative $\mathrm{LN}$ cases. (Alharazy et al., 2015) found that there were significant differences between the active and inactive LN groups with regard to renal SLEDAI.

According to the WHO grading scheme for $\mathrm{LN}$, most of our cases had class IV LN (60.60\%) and only one (3.03\%) had advanced sclerotic lupus nephritis with activity index ranged from 1 to $15 / 24$ and chronicity index ranged from 1 to $10 / 12$.

The mean value of RRI in group I was statistically significantly higher than that in group II and group III with no significant difference between group II and group III. Out of 33 cases of LN cases, 6 patients had RRI of 0.7 and above giving a percentage of $18.18 \%$. This finding was matching the study carried by (Conti et al., 2014), where a higher mean value of RRI was calculated in $L N$ patients than in SLE without renal affection than in normal subjects.

There was a statistically significant difference between the mean values of creatinine among different $L N$ classes with higher levels in class IV LN, this was matching with most data (Appel and D'Agati, 2007; Markowitz and D'Agati, 2009). In addition serum creatinine and blood urea were significantly correlated with renal biopsy chronicity index. This matched with the study carried by Satirapoj et al. (2015).

Conti et al. (2014) also recorded RRI>0.7 only in patients with renal SLE suggesting a correlation of a pathologic RRI with glomerular lesions and histological classes. Platt et al. (1997) found RRI $>0.7$ in $29 \%$ of 34 $\mathrm{LN}$ patients in a trial to correlate a pathologic RRI and chronicity index to poorer renal outcome after 1-y followup. Gao et al. (2013) retrospectively reviewed 24 LN patients and observed a significantly higher RRI in patients with moderate renal cortical fibrosis than in those with mild fibrosis.

In our study, we found that RRI significantly correlated with age, $\mathrm{Hb}, \mathrm{MBP}$, FBS, blood urea, serum creatinine, eGFR, uACR, ESR,$C 3$ and chronicity index in renal biopsy. A significant correlation between RRI and renal function evaluated by urea, creatinine and eGFR in kidney diseases irrespective of etiologies has been demonstrated in many studies (Sugiura and Wada, 2011; Mostbeck et al., 1991; Ikee et al., 2005; Sugiura et al., 2004); others also significantly correlated RRI with age, systolic blood pressure and proteinuria in CKD. Hypertension and age are well-known parameters that influence renal resistance and vascular compliance
(Parolini et al., 2009; Sugiura and Wada, 2009).

While correlation with proteinuria may be due to the fact that almost all of the pathological RRI are detected in classes III, IV and V which arecharacterized by overt proteinuria, also proteinuria is both a marker for greater degree of renal damage as well as an independent risk factor for CKD progression. Moreover, the RRI has emerged as an independent risk factor for worsening of renal function in large follow-up studies on different renal diseases: renal survival rate was significantly lower in patients with a baseline RRI 0.7 (Sugiura and Wada, 2011; Sugiura and Wada, 2009).

On the other hand RRI did not significantly correlated with other parameters as serum albumin, anti-ds DNA, C4, SLEDAI scores, SLICCRAS score and activity index in renal biopsy, that in addition we could not find any significant correlation between RRI and any specific histological class although we could observe a lower mean of RRI in class II in comparison to other classes, which may aid in assessing the role of RRI in differentiating between different classes of $L N$ with different outcomes and prognosis. The non-significance with SLEDAI score is not surprising. These are composite indices including items on involvement of different organs.

From our study we found that LN patients with RRI of 0.7 or more had higher age, higher mean blood urea and serum creatinine level and higher mean value of chronicity index of renal biopsy but no statistical significant difference was observed between renal biopsy classes and RRI of 0.7 or more.

Several studies evaluated the role of RRI in LN, the first one by Platt et al. (1997) who described a correlation between a pathologic RRI and creatinine level, histologic chronicity index and presence of interstitial disease only while therewas an absent correlation between RRI and uACR, anti dsDNA, C3, C4, biopsy classes, activity index or total biopsy score. Moreover, a pathologic RRI and chronicity index were related to poorer renal outcome after $1 \mathrm{yr}$ follow-up (doubling serum creatinine and/or need of RRT). Patients with an initial RRI of 0.70 or higher showed a worse outcome, independent of initial eGFR and they concluded that RRI and not the serum creatinine is significantly higher in those with poorer outcomes.

Later on, Wang et al. (2007) in his study on 44 patients with class IV LN, showed that the chronicity index of renal biopsy was significantly higher in patients with increased RRI. On the other hand when population study is divided according to immune suppression response, it was proven that increased RRI was significantly associated with an increased likelihood of poor response to treatment.

Gao et al. (2013) retrospectively reviewed 24 LN patients and observed a significantly higher RRI at the level of main renal artery (and not as in our study and other studies at level of interlobar arteries) in patients 
with moderate renal cortical fibrosis than in those with mild fibrosis, with no marked difference at the level of interlobar arteries. It is worth saying there was a significant difference between both groups regarding serum creatinine. This study focused principally on the early stages of renal hemodynamic disturbances to grade renal cortical fibrosis. One of limitations of this study in addition to assessing patients retrospectively, the renal biopsy specimens when evaluating chronic irreversible changes only accounted on interstitial fibrosis and tubular atrophy while ignoring the other 2 parameters glomerulosclerosis and arteriosclerosis which are major determinants of intra-renal hemodynamic change (Bude and Rubin, 1999).

Conti (2014) as others have found that the RRI to be positively correlated only with creatinine and BUN and negatively correlated with eGFR, in addition out of 5 patients with pathological $R R I>0.7$ in the 42 patients $L N$ enrolled, 4 of them belong to class IV LN and 1 belonging to class III.

In addition, in controversy to other studies, Conti et al. (2014) found that SLE patients with RRI 0.7 had significantly higher mean activity index values with no significant correlation with the chronicity index. This may be in part due prevalence of severe glomerular histologic features (glomerular synechiae and glomerular cellularfibrotic crescent formation), which are indicative of a worst prognosis in his studying sample, nevertheless the relatively low chronicity indices scores of his specimens. A similar observation was detected in our study where in both of the 2 patients having crescents (100\%), RRI $>0.7$ was calculated.

Study done by Gigante et al. (2016) on 100 biopsy proven GN including about $30 \mathrm{LN}$ patients founded a significant correlation between RRI and age, serum creatinine, eGFR, $\%$ of glomerulosclerosis and of crescents and degree of arteriosclerosis .

From the study of ROC curve of Doppler-Based Renal Resistive index, we suggested that 0.70 threshold was better than 0.65 cut-off to predict renal biopsy chronicity index, although it has a higher sensitivity but with lower specificity and positive predictive value.

Chronicity index $(\mathrm{Cl})$ score had been found to be important predictor of long-term outcome in LN (Magil et al., 1988; Esdaile et al., 1991). It was demonstrated that tubulointerstitial lesions as the major locations of injury for determining prognosis in LN (Austin et al., 1994). The severity of interstitial disease correlated with the degree of renal insufficiency and was a valuable marker for progressive deterioration in renal function (Schwartz et al., 1992). Therefore, the predictive value of RRI is likely to result from the correlation of $\mathrm{RRI}$ with $\mathrm{Cl}$ and tubulointerstitial changes.

Limitations of kidney biopsy are a result of its invasive nature, which prevents periodic sampling especially in the clinical setting of inactive lupus and renal symptoms, and its small sample size that might not reflect the disease in the entire kidney. As new technologies for the earlier detection of kidney injury in LN such as biomarkers are emerging, renal sonography should provide complementary information (Manoharan and Madaio, 2010; Zhang et al., 2008).

Thus from the results of our study, we could conclude that renal Doppler is a useful non-invasive technique that can be used to evaluate chronicity of renal affection in SLE, and therefore justifying aggressive immune suppression, but further follow-up studies are needed to evaluate its role in predicting response to treatment. The strength of the present study is the homogeneity of the population evaluated and the inclusion of patients in different histologic classes of $L N$ taking into account both glomerular and non-glomerular lesions.

\section{REFERENCES}

Aikimbaev KS, Canataroglu A, Ozbek S, Usal A, 2001. Renal vascular resistance in progressive systemic sclerosis: evaluation with duplex Doppler ultrasound. Angiology, 52: 697-701.

Alharazy S, Kong NC, Mohd M, Shah SA, Ba'in A, Abdul Gafor AH, 2015. Urine monocyte chemoattractant protein-1 and lupus nephritis disease activity: preliminary report of a prospective longitudinal study. Autoimmune Dis, 1-13.

Appel GB, D'Agati VD, 2007. Lupus nephritis-pathology and pathogenesis. In: Dubois' Lupus erythematosus. Wallace DJ, Hahn BH (Eds), Philadelphia: Lippincott Williams \& Wilkins, pp: 1094-1112.

Austin HA, Boumpas DT, Vaughan EM, Balow JE, 1994. Predicting renal outcomes in severe lupus nephritis: Contributions of clinical and histologic data. Kidney Int, 45: $544-550$.

Bain BJ, Lewis SM and Bates I, 2006. Basic haematological techniques. In: Dacie and Lewis practical haematology, Lewis SM, Bain BJ, Bates I (Eds). 10 ${ }^{\text {th }}$ Ed. Philadelphia: Churchill Livingstone, pp: 25-54.

Ballow JE, Buompas DT, Fester BJ, Austin HA, 1996. Management of lupus nephritis. Kidney Int, 53: 88-92.

Bertsias G, Cervera R, Boumpas DT, 2012. Systemic lupus erythematosus :pathogenesis and clinical features. In: EULAR textbook on rheumatic diseases, Bijlsma J (ed) London: BMJ Group, pp: 476-505.

Birmingham DJ, Rovin $\mathrm{BH}$, Shidham G, Bissell M, Nagaraja HN, Hebert LA, 2008. Relationship between albuminuria and total proteinuria in systemic lupus erythematosus nephritis: diagnostic and therapeutic implications. Clin J Am Soc Nephrol, 3(4): 1028-1033.

Boumpas DT, Austin HA, Fessler BJ, Balow JE, Klippel JH, Lockshin MD, 1995. Systemic lupus erythematosus: emerging concepts. Part 1. Renal, neuropsychiatric, cardiovascular, pulmonary and hematologic disease. Ann Intern Med, 122: 940-950.

Bude RO, Rubin JM, 1999. Relationship between the resistive index and vascular compliance and resistance. Radiology, 211: 411-417.

Burtis CA, Ashwood ER, Bruns DE, 2006. Tietz Textbook of clinical chemistry and molecular diagnostics. $4^{\text {th }}$ Ed, St Louis, Elsevier Saunders Company, PP: 548 (albumin), 797-802 (urea, creatinine), 808-12 (urine), 868-71 (glucose).

Cervera R, Khamashta MA, Font J, Sebastiani GD, Gil A, Lavilla P, 2003. Morbidity and mortality in systemic lupus erythematosus during a 10-year period: a comparison of early and late manifestations in a cohort of 1,000 patients. Medicine, 82(5): 299-308.

Chang E, Abrahamowicz M, Ferland D, Fortin PR, 2002. Comparison of the responsiveness of lupus disease activity measures to changes in systemic lupus erythematosus activity relevant to patients and physicians. J Clin Epidemiol, 55(5): 488-497.

Chen Q, He F, Feng X, Luo Z, Zhang J, Zhang L, 2014. Correlation of Doppler parameters with renal pathology: A study of 992 patients. Exp Ther Med, 7: 439-442.

Cholongitas E, Shusang V, Marelli L, Nair D, Thomas M, Patch D, 2007. Renal function assessment in cirrhosis-difficulties and 
alternative measurements. Aliment Pharmacol Ther, 26: 969-978.

Conti F, Ceccarelli F, Gigante A, Barbano B, Perricone C, Massaro L, 2014. Ultrasonographic evaluation of renal resistive index in patients with lupus nephritis: correlation with histologic findings. Ultrasound Med Biol, 40(11): 2573-1580.

Corapi KM, Dooley MA and Pendergraft WF, 2015. Comparison and evaluation of lupus nephritis response criteria in lupus activity indices and clinical trials. Arthritis Res Ther, 15; 17(110): 1-13.

Crutchley TA, Pearce JD, Craven TE, Stafford JM, Edwards MS, Hansen KJ, 2009. Clinical utility of the resistive index in atherosclerotic renovascular disease. J Vasc Surg, 49: 148-155.

D’Agati V, 1998. Renal disease in systermic lupus erythematosus, mixed connective tissue disease, Sjogren's syndrome, and rheumatoid arthritis. In: Pathology of the kidney. Jennette C, Ison JL, Schwartz MM, Silva FG (Eds). $5^{\text {th }}$ Ed. Philadelphia; Lippincott-Raven Publishers, pp: 541-624.

Edworthy SM, 2001. Clinical manifestations of systemic lupus erythematosis. In: Textbook of rheumatology, Kelly WN, Ruddy S and Sledye CB $\left(6^{\text {th }} \mathrm{Ed}\right)$. Philadelphia: WB Saunders, pp: 1105-1118.

El-Shehaby A, Darweesh H, El-Khatib M, Momtaz M, Marzouk S, ElShaarawy N, 2011. Correlations of urinary biomarkers, TNF-like weak inducer of apoptosis (TWEAK), osteoprotegerin (OPG), monocyte chemoattractant protein-1 (MCP-1), and IL-8 with lupus nephritis. J Clin Immunol, 31(5): 848-856.

Esdaile JM, Federgreens W, Quintal H, Suisse S, Hayslett JP, Kashgarian M, 1991. Predictors of one year outcome in lupus nephritis: the importance of renal biopsy. Q J Med, 81: 907-918.

Farid EM, Hassan AB, Abalkhail AA, El-Agroudy AE, Arrayed SA, AlGhareeb SM, 2013. Immunological aspects of biopsy-proven lupus nephritis in Bahraini patients with systemic lupus erythematosus. Saudi J Kidney Dis Transpl, 24(6): 1271-1279.

Fouda N, Abaza N, El-Hilaly R, El Said HW, EL-kabarity RH, 2012. Evaluation of visfatin in patients with systemic lupus erythematosus: Correlation with disease activity and lupus nephritis. Egyptian Rheumatol, 34: 9-17.

Gao J, Chevalier J, Auh YH, Rubin JM, Wang H, Sun LN, 2013. Correlation between Doppler parameters and renal cortical fibrosis in lupus nephritis: A preliminary observation. Ultrasound Med Biol, 39: 275-282.

Gigante A, Barbano B, Di Mario F, Rosato E, Simonelli M, Rocca AR, 2016. Renal parenchymal resistance in patients with biopsy proven glomerulonephritis: Correlation with histological findings. Int J Immunopathol Pharmacology, 1: 1-6.

Granata A, Andrulli S, Bigi MQ, Pozzoni P, Fiorini F, Logias F, Fiquera M, Basile A, Fiore CE, 2009. Predictive role of duplex Doppler ultrasonography in the diagnosis of acute renal obstruction in patients with unilateral renal colic. Clin Nephrol, 71(6): 680-686.

Granata A, Zanoli L, Clementi S, Fatazzo P, Di Nicolo P, Fiorini F, 2014. Resistive intra renal index. Myth or reality. Br J Radiol, 87: 1-7.

Hahn BH, McMahan M, Wilkinson A, Wallace WD, Daikh DI, FitzGerald $\mathrm{J}$, 2012. American College of Rheumatology Guidelines for Screening, Case Definition, Treatment and Management of Lupus nephritis. Arthritis Care Res, 64(6): 797-808.

Hanamura K, Tojo A, Kinugasa S, Asaba K, Fujita T, 2012. The resistive index is a marker of renal function, pathology, prognosis, and responsiveness to steroid therapy in chronic kidney disease patients. Int J Nephrol, 1-9.

Ikee R, Kobayashi S, Hemmi N, Imakiire T, Kikuchi Y, Moriya H, 2005. Correlation between the resistive index by Doppler ultrasound and kidney function and histology. Am J Kidney Dis, 46: 603-609.

Isenberg DA, Rahman A, Allen E, Farewell V, Akil M, Bruce IN, 2005. Disease activity index for patients with SLE. Rheumatology, 44(7): 902-906.

Jane C, David J, 2003. Lupus nephritis. Medicine, 31(7): 101-104.

Kalantarinia K, 2009. Novel imaging techniques in acute kidney injury. Curr Drug Targets, 10(12): 1184-1189.

Korbet SM, 2002. Percutaneous kidney biopsy. Semin Nephrol, 22: 254-267.

Krumme B, 2006. Renal Doppler sonography-Update in clinical nephrology. Nephron Clin Pract, 103c: 24-28.

Lawrence RC, Helmick CG, Arnett FC, Deyo RA, Felson DT, Giannini $\mathrm{EH}, 1998$. Estimates of the prevalence of arthritis and selected musculoskeletal disorders in the United States. Arthritis Rheum, 41(5): 778-799.

Le Dorze M, Bougle' A, Deruddre S, Duranteau J, 2012. Renal Doppler ultrasound: a new tool to assess renal perfusion in critical illness. Shock, 37: 360-365.

Lee $\mathrm{YH}$, Woo JH, Choi SJ, Ji JD, Song GG, 2010. Induction and maintenance therapy for lupus nephritis: a systematic review and meta-analysis. Lupus, 19: 703-710.

Linnik MD, Hu JZ, Heilbrunn KR, Strand V, Hurley FL, Joh T, 2005. Relationship between anti-double-stranded DNA antibodies and exacerbation of renal disease in patients with systemic lupus erythematosus. Arthritis Rheum, 52(4): 1129-1137.

Lubas A, Kade G, Niemczyk S, 2014. Renal resistive index as a marker of vascular damage in cardiovascular diseases. Int Urol Nephrol, 46: 395-402.

Magil AB, Puterman ML, Ballon HS, Chan V, Lirenman DS, Rae A, 1988. Prognostic factors in diffuse proliferative lupus glomerulonephritis. Kidney Int, 34(4): 511-517.

Manoharan A, Madaio M, 2010. Biomarkers in lupus nephritis. Rheum Dis Clin N Am, 36: 131-143.

Markowitz GS1, D'Agati VD, 2009. Classification of lupus nephritis. Curr Opin Nephrol Hypertens, 18: 220-225.

Moc CC, Lau CS, 2003. Pathogenesis of systemic lupus erythematosus. J Clin Pathol, 56: 481-490.

Mok CC, 2010. Biomarkers for lupus nephritis: a critical appraisal. J Biomed Biotechnol, 1-11.

Mok CC, Birmingham DJ, Ho LY, Hebert LA, Rovin BH, 2013. Highsensitivity $\mathrm{C}$-reactive protein, disease activity and cardiovascular risk factors in systemic lupus erythematosus. Arthritis Care Res, 65(3): 441-447.

Mostbeck G, Kain R, Mallek R, 1991. Duplex Doppler sonography in renal parenchymal disease; histo-pathologic correlation. J Ultrasound Med, 10: 189-194.

Najafi CC, Korbert SM, Lewis EJ, Schwartz MM, Reichlin M, Evanz J, 2001. Significance of histological patterns of glomerular injury upon long term prognosis in severe lupus glomerulonephritis. Kidney Int, 59: 2156-2163.

Narayanan CK, Marwaha CV, Shanmuganandan CK, Shankar GS, 2010. Correlation between Systemic Lupus Erythematosus Disease Activity Index, C3, C4 and Anti-dsDNA Antibodies. MJAFI, 66: 102107.

Ozbek SS, Buyukberber S Tolunay O, Erden MI, Aytac SK, Olmez U, 1995. Image-directed color Doppler ultrasonography of kidney in systemic lupus nephritis. J Clin Ultrasound, 23: 17-20.

Parolini C, Noce A, Staffolani E, Giarrizzo GF, Costanzi S, Splendiani $G$, 2009. Renal resistive index and long-termoutcome in chronic nephropathies. Radiology, 252: 888-896.

Patriquin HB, O'Regan S, Robitaille P, Paltiel H, 1989. Hemolyticuremic syndrome: intrarenal arterial Doppler patterns as a useful guide to therapy. Radiology, 172: 625-628.

Petri M, Kasitanon N, Lee SS, Link K, Magder L, Bae SC, 2008. Systemic lupus international collaborating clinics renal activity/response exercise. Development of a renal activity score and renal response index. Arthritis Rheum, 58(6): 1784-1788.

Petri M, Orbai AM, Alarcon GS, Gordon C, Merrill JT, Fortin PR, 2012. Derivation and validation of the Systemic Lupus International Collabor Clinics classification criteria for systemic lupus erythematosus. Arthritis Rheum, 64(8): 2677-2686.

Pitashny M, Schwartz N, Qing X, Hojaili B, Aranow C, Mackay M, 2007. Urinary lipocalin-2 is associated with renal disease activity in human lupus nephritis. Arthritis Rheum, 56: 1894-1903.

Platt JF, Ellis JH, Rubin JM, Di Pietro MA, Sedman AB, 1990. Intrarenal arterial Doppler sonography in patients with non-obstructive renal disease: Correlation of resistive index with biopsy findings. Am J Roentgenol, 154: 1223-1227.

Platt JF, Rubin JM, Ellis JH, 1997. Lupus nephritis: predictive value of conventional and Doppler US and comparison with serologic and biopsy parameters. Radiology, 7: 203: 82-86.

Radermacher J, Ellis S, Haller H, 2002. Renal resistance index and progression of renal disease. Hypertension, 39: 699-703.

Radermacher J, Mengel M, Ellis S, Stuht S, Hiss M, Schwarz A, 2003. The renal arterial resistance index and renal allograft survival. $N$ Engl 
J Med, 349: 115-124.

Rahman A, Isenberg DA, 2008. Systemic lupus erythematosus. N Engl J Med, 358(9): 929-939.

Rauta TP, Patila TB, Khota RS, Sargarb KM, Patila MB, Bansoda YV, 2012. Clinical profile of diabetic nephropathy and correlation with intra-renal resistivity index by Duplex ultrasonography. World $\mathrm{J}$ Nephrol Urol, 1(4-5): 107-114.

Rosato E, Gigante A, Barbano B, Cianci R, Molinaro I, Rossi C, 2012. Intrarenal hemodynamic parameters correlate with glomerular filtration rate and digital micro-vascular damage in patients with systemic sclerosis. Semin Arthritis Rheum, 41: 815-821.

Satirapoj B, Tasanavipas P, Supasyndh O, 2015. Clinico-pathological correlation in Asian patients with biopsy-proven lupus nephritis. Int $\mathrm{J}$ Nephrol, 1-6.

Satirapoj B, Wongchinsri J, Youngprang N, Laonapaporn B, Chitrada T, Lapkittichareonchai S, Patumanon J, 2007. Predictors of renal involvement in patients with systemic lupus erythematosus. Asian Pac J Allergy Immunol, 25(1): 17-25.

Schwartz MM, Lan SP, Bernstein J, 1992. Role of pathology in indices in the management of severe lupus glomerulonephritis. Kidney Int, 42: 743-748.

Sox HC, Liang $M H$, 1986. The erythrocyte sedimentation rate: Guidelines for rational use. Ann Intern Med, 104: 515-523.

Stanley JH, Cornella R, Loevinger E, 1984. Sonography of systemic lupus nephritis. Am J Roentgenol, 142: 1165-1169.

Stojan G, Fang H, Magder L, Petri M, 2013. Erythrocyte sedimentation rate is a predictor of renal and overall SLE disease activity. Lupus, 22(8): 827-834.

Sugiura T, Nakamori A, Wada A, Fukuhara Y, 2004. Evaluation of tubulointerstitial injury by Doppler ultrasonography in glomerular diseases. Clin Nephrol, 61(2): 119-126.

Sugiura T, Wada A, 2009. Resistive index predicts renal prognosis in chronic kidney disease. Nephrol Dial Transplant, 24(9): 2780-2785.

Sugiura T, Wada A, 2011. Resistive index predicts renal prognosis in chronic kidney disease: Results of a 4-y follow-up. Clin Exp Nephrol, 15: 114-120.

Uramoto KM, Michet CJJ, Thumboo J, Sunko J, O'Fallon WM, Gabriel SE, 1999. Trends in the incidence and mortality of systemic lupus erythematosus. Arthritis Rheum, 42: 46-50.

Valesini G, Conti F, 2011. The persistent challenge of lupus nephritis. Clin Rev Allergy Immunol, 40: 135-137.

Viazzi F, Leoncini G, Derchi LE, Pontremoli R, 2014. Ultrasound Doppler renal resistive index: a useful tool for the management of the hypertensive patient. J Hypertens, 32: 149-153.

Wang CL, Shu KH, Lan JL, Cheng CH, Wu MJ, Chen CH, 2007. Duplex Doppler sonography to predict response to therapy in active lupus nephritis. Kuang Tien Med J, 2(1): 21-28.

Weening JJ, D'Agati VD, Schwartz MM, Seshan SV, Alpers CE, Appel $\mathrm{GB}, 2004$. The classification of glomerulonephritis in systemic lupus erythematosus revisited. Kidney Int, 65(2): 521-530.

Yip J, Aghdassi E, Su J, Lou W, Reich H, Bargman J, 2010. Serum albumin as a marker for disease activity in patients with systemic lupus erythematosus. J Rheumatol, 37(8): 1667-1672.

Zabaleta-Lans ME, Munoz LE, Tapanes FJ, Vargas-Arenas RE, Daboin I, Barrios Y, 2006. Further description of early clinically silent lupus nephritis. Lupus, 15:845-851.

Zappitelli M, Duffy CM, Bernard C, Gupta IR, 2008. Evaluation of activity, chronicity and tubule-interstitial indices for childhood lupus nephritis. Pediatr Nephrol, 23(1): 83-91.

Zhang XL, Jin M, Wu HF, Nadasdy T, Nadasdy G, Harris N, 2008. Biomarkers of lupus nephritis determined by serial urine proteomics. Kidney Int, 74: 799-807.

Zweig MH, Campbell G, 1993. Receiver-operating characteristic (ROC) plots: a fundamental evaluation tool in clinical medicine. Clin Chem, 39(4): 561-577.
Citation: El-Wakil H, Khali E, M aharem D, Mahena E, Hasab M, 2017. Renal arterial resistive index in Egyptian patients with lupus nephritis: Correlation with disease activity and biopsy parameters. Int Res J Med Med Sci, 6(1): 18-34. 